\title{
ON THE UNIFORM KADEC-KLEE PROPERTY WITH RESPECT TO CONVERGENCE IN MEASURE
}

\author{
F. A. SUKOCHEV \\ (Received 25 April 1993; revised 8 October 1993)
}

Communicated by P. G. Dodds

\begin{abstract}
Let $E(0, \infty)$ be a separable symmetric function space, let $M$ be a semifinite von Neumann algebra with normal faithful semifinite trace $\mu$, and let $E(M, \mu)$ be the symmetric operator space associated with $E(0, \infty)$. If $E(0, \infty)$ has the uniform Kadec-Klee property with respect to convergence in measure then $E(M, \mu)$ also has this property. In particular, if $L_{\Phi}(0, \infty)\left(\Lambda_{\varphi}(0, \infty)\right)$ is a separable Orlicz (Lorentz) space then $L_{\Phi}(M, \mu)\left(\Lambda_{\varphi}(M, \mu)\right)$ has the uniform Kadec-Klee property with respect to convergence in measure. It is established also that $E(0, \infty)$ has the uniform Kadec-Klee property with respect to convergence in measure on sets of finite measure if and only if the norm of $E(0, \infty)$ satisfies $\mathrm{G}$. Birkhoff's condition of uniform monotonicity.
\end{abstract}

1991 Mathematics subject classification (Amer. Math. Soc.): primary 46B20; secondary 46E30, 46L50.

\section{Introduction}

Let $\left(X,\|\cdot\|_{x}\right)$ be a Banach space, and let $\tau$ be a topological vector space topology on $X$ that is weaker than the norm topology. The space $\left(X,\|\cdot\|_{x}\right)$ is said to have the uniform Kadec-Klee property with respect to $\tau$ (notation $X \in\left(\mathrm{UH}_{\tau}\right)$ ) if for all $\epsilon>0$ there exists $\delta(\epsilon)>0$ such that for every sequence $\left(x_{n}\right) \subset X$ with $\left\|x_{n}\right\|_{x}=1$, $\left\|x_{n}-x_{m}\right\|_{x}>\epsilon(m \neq n)$ and with limit $x$ in the topology $\tau$, we have $\|x\|_{x}<1-\delta(\epsilon)$.

We will consider the following cases:

(1) $X$ is a Banach space with a Schauder basis $\left(e_{n}\right), \tau=\sigma(X, \Gamma)$ where $\Gamma=\left[e_{n}^{*}\right]$;

(2) $X$ is a symmetric function space $E(0, \infty), \tau=$ convergence locally in measure and

(3) $X=E(0, \infty)(X=E(M, \mu)), \tau=$ convergence in Lebesgue measure $m$ ( $\tau=$ convergence in the measure topology on the set of all $\mu$-measurable operators (see

(C) 1995 Australian Mathematical Society $0263-6115 / 95 \$ A 2.00+0.00$ 
[5])). We will denote the $\left(\mathrm{UH}_{\tau}\right)$-property by $\left(\mathrm{UH}_{\Gamma}\right)$ in the first case; by $(\mathrm{UHlm})$ in the second and by $(\mathrm{UHm})((\mathrm{UH} \mu))$ in the third.

See $[8,10,11]$ for general information concerning Banach spaces and symmetric function spaces. For relevant terminology from the theory of the von Neumann algebras we refer to [17], and for the theory of non-commutative integration we refer to [5].

\section{Property $\left(\mathrm{UH}_{\Gamma}\right)$ in spaces with Schauder basis}

Let $(X,\|\cdot\|)$ be a Banach space with a Schauder basis $\left(e_{n}\right)$. Throughout this section $\left(e_{n}^{*}\right)$ are the bi-orthogonal functionals associated with $\left(e_{n}\right)$, and $P_{n}$ are the projections onto $\left[e_{k}\right]_{k=1}^{n}$ with kernel $\left[e_{k}\right]_{k=n+1}^{\infty}$; that is for every $x=\sum_{k=1}^{\infty} e_{k}^{*}(x) e_{k}$, we have $P_{n} x=\sum_{k=1}^{n} e_{k}^{*}(x) e_{k}$.

The basis $\left(e_{n}\right)$ is said to satisfy the condition $(C)$ if for every $c>0$ there exists $\delta=\delta(c)>0$ such that for every $x \in X$ and for each integer $n$ it follows from the conditions $\left\|P_{n} x\right\|=1$ and $\left\|\left(I-P_{n}\right) x\right\| \geq c$ that

$$
\|x\| \geq 1+\delta .
$$

The Theorem below is due (in implicit form) to D. van Dulst and V. de Valk [4] for the case when $X$ is an Orlicz sequence space; however, it is also true more generally. We omit the proof and refer to [4, Proposition 3].

THEOREM 1. If a basis $\left(e_{n}\right)$ satisfies the condition $(C)$ then $X \in\left(\mathrm{UH}_{\Gamma}\right)$, where $\Gamma=\left[e_{n}^{*}\right]_{n=1}^{\infty}$.

It is well known that every Banach space with an unconditional basis $\left(e_{n}\right)$, whose unconditional constant is equal to 1 , is a Banach lattice when the order is defined by $\sum_{n=1}^{\infty} a_{n} e_{n} \geq 0$ if and only if $a_{n} \geq 0$ for all $n$. A Banach lattice $\left(X,\|\cdot\|_{x}\right)$ is called a UMB-lattice (notation: $\left(X,\|\cdot\|_{x}\right) \in(\mathrm{UMB})$ ) if its norm satisfies $\mathrm{G}$. Birkhoff's condition of uniform monotonicity; that is for all $\epsilon>0$ there exists $\delta(\epsilon)>0$ such that if $f, g \in X, f \geq 0, g \geq 0,\|f\|_{x}=1$ and $\|f+g\|_{x} \leq 1+\delta(\epsilon)$ then $\|g\|_{x} \leq \epsilon$ (see [2]). In addition, if we suppose that $f \wedge g=0$, then $X$ is said to have the property (UMBd) (notation: $\left(X,\|\cdot\|_{x}\right) \in(\mathrm{UMBd})$ ). We remark that the property (UMBd) coincides with the property $(C)$ which is considered in [3]. Evidently, if $\left(X,\|\cdot\|_{x}\right) \in(\mathrm{UMBd})$ and the order on $X$ is defined by the unconditional basis $\left(e_{n}\right)$, then $\left(e_{n}\right)$ satisfies condition $(C)$. So, we have the following

COROLlaRY 1. (See also [3]). Let $X$ be a Banach lattice whose order is induced by the 1-unconditional basis $\left(e_{n}\right)$. If $\left(X,\|\cdot\|_{x}\right) \in(\mathrm{UMBd})$ then $X \in\left(\mathrm{UH}_{\Gamma}\right)$. 
The next theorem shows that in the preceding corollary the condition $\left(X,\|\cdot\|_{x}\right) \epsilon$ (UMBd) is also necessary if basis $\left(e_{n}\right)$ is symmetric.

THEOREM 2. Let X be a Banach lattice whose order is induced by the 1-symmetric basis $\left(e_{n}\right)$. Then the following conditions are equivalent:

(i) $\left(X,\|\cdot\|_{x}\right) \in(\mathrm{UMB})$;

(ii) $\left(X,\|\cdot\|_{x}\right) \in(\mathrm{UMBd})$;

(iii) $\left(X,\|\cdot\|_{x}\right) \in\left(\mathrm{UH}_{\Gamma}\right)$.

PROOF. The implication (ii) $\rightarrow$ (iii) follows from Corollary 1. The implication (i) $\rightarrow$ (ii) is obvious.

Before proving the implication (iii) $\rightarrow$ (i) of the theorem, we recall the following facts. Let $a=\left(a_{i}\right), b=\left(b_{i}\right) \in c_{0}$, let $a^{*}=\left(a_{i}^{*}\right), b^{*}=\left(b_{i}^{*}\right)$ be the sequences $\left(\left|a_{i}\right|\right)$, $\left(\left|b_{i}\right|\right)$ arranged in non-increasing order. We say that $a$ is weakly submajorized by $b$ and write $a \prec_{w} b$ if $\sum_{i=1}^{k} a_{i}^{*} \leq \sum_{i=1}^{k} b_{i}^{*}$, for $k=1,2,3, \ldots$ It is well known that if $a \prec_{w} b, b=\left(b_{i}\right) \in X$ (we identify $b=\sum_{i} b_{i} e_{i}$ with $\left(b_{i}\right)$ ) then $a \in X$ and $\|a\|_{x} \leq\|b\|_{x}$.

Divide the set $N$ into two disjoint subsets $A$ and $B$ with card $A=$ card $B=\infty$. Let $\pi_{1}: N \rightarrow A, \pi_{2}: N \rightarrow B$ be arbitrary injections. Put $a^{\prime}=\sum_{k} e_{k}^{*}(a) e_{\pi_{1}(k)}$, $b^{\prime}=\sum_{k} e_{k}^{*}(b) e_{\pi_{2}(k)}$. It is clear that $a^{*}=\left(a^{\prime}\right)^{*}, b^{*}=\left(b^{\prime}\right)^{*}$.

The proof of the following lemma is straightforward. The details are therefore omitted. It should be pointed out that the lemma which follows is a special case of Lemma 3 below.

LemMA 1. Let $a, b \in X, a \geq 0, b \geq 0$. Then $a^{\prime}+b^{\prime} \prec_{w} a+b$ and therefore $\left\|a^{\prime}+b^{\prime}\right\|_{x} \leq\|a+b\|_{x}$.

We remark that it follows immediately from Lemma 1 that (ii) implies (i).

Let us continue the proof of Theorem 2. Assume that $X \in\left(\mathrm{UH}_{\Gamma}\right)$ but that the condition of uniform monotonicity fails to hold for $X$. Then there exist $\epsilon>0$; $\left(x_{n}\right),\left(y_{n}\right) \subset X, x_{n}, y_{n} \geq 0$, such that $\left\|x_{n}\right\|_{x}=1,\left\|y_{n}\right\|_{x} \geq \epsilon$, and $\left\|x_{n}+y_{n}\right\|_{x}<1+n^{-1}$. Fix the integer $n$ and divide the set $N$ into an infinite family of disjoint subsets $C_{k}$ with card $C_{k}=\infty, k=0,1,2, \ldots$ Let $\pi_{k}: N \rightarrow C_{k}$ be arbitrary bijections. Put $a=\sum_{i} e_{i}^{*}\left(x_{n}\right) e_{\pi_{0}(i)}, b_{k}=\sum_{i} e_{i}^{*}\left(y_{n}\right) e_{\pi_{k}(i)}$. It is clear that $\left(a+b_{k}\right)^{*}=\left(a+b_{m}\right)^{*}$ for $k, m=1,2, \ldots$, and $\sigma(X, \Gamma)-\lim _{k} b_{k}=0$. Using Lemma 1 , we have

$$
1 \leq\left\|a+b_{k}\right\|_{x} \leq\left\|x_{n}+y_{n}\right\|_{x}<1+n^{-1} .
$$

Therefore $\left\|\alpha^{-1}\left(a+b_{k}\right)\right\|_{x}=1,\left\|\alpha^{-1}\left(b_{k}-b_{m}\right)\right\|_{x} \geq \alpha^{-1}\left\|b_{k}\right\|_{x}=\alpha^{-1}\left\|y_{n}\right\|_{x} \geq \alpha^{-1} \epsilon \geq$ $2^{-1} \epsilon$ and $\sigma(X, \Gamma)-\lim _{k}\left(\alpha^{-1}\left(a+b_{k}\right)\right)=\alpha^{-1} a$, where $\alpha=\left\|a+b_{k}\right\|_{x}$. Since $X \in\left(\mathrm{UH}_{\Gamma}\right)$ we have $\left\|\alpha^{-1} a\right\|_{x}<1-\delta\left(2^{-1} \epsilon\right)$. Using (1), it now follows that

$$
1=\left\|x_{n}\right\|_{x}=\|a\|<\left(1+n^{-1}\right)\left(1-\delta\left(2^{-1} \epsilon\right)\right)
$$


for all $n=1,2, \ldots$ This contradicts the fact that $\delta\left(2^{-1} \epsilon\right)>0$ and thus completes the proof of Theorem 2.

REMARK. The equivalence of (ii) and (iii) has been established also in [6].

\section{2. (UHlm)-property for the symmetric function spaces $E(0, \infty)$}

Throughout this section $E(0, \infty)$ is a separable symmetric function space. It is well known (see [11]), that every space $X$ with a symmetric basis $\left(e_{n}\right)$ is a symmetric function space on an infinite discrete measure space $\Omega$ in which the mass of every point is one. In this context $\sigma(X, \Gamma)$-convergence coincides with convergence in measure on sets of finite measure on the unit sphere of $X$. The following question therefore naturally arises from Theorem 2: are the properties (UHIm)and (UMB) equivalent in $E(0, \infty)$ ? The theorem below gives a positive answer to this question.

THEOREM 3. For a separable symmetric space $(E(0, \infty),\|\cdot\|)$ the following conditions are equivalent:

(i) $(E(0, \infty),\|\cdot\| \in(\mathrm{UMB})$;

(ii) $(E(0, \infty),\|\cdot\| \in(\mathrm{UMBd})$;

(iii) $(E(0, \infty),\|\cdot\| \in(\mathrm{UHlm})$.

PROOF. Assertion (ii) is a consequence of assertion (i). It follows from [3], Theorem 3.3 , that assertion (iii) is a consequence of (ii). The implication (iii) $\rightarrow$ (i) is proved exactly in the same way as in Theorem 2. Instead of relation $a \prec_{w} b$ between sequences $a=\left(a_{n}\right)$ and $b=\left(b_{n}\right)$ we consider the relation $f \prec g$ between functions $f, g \in L_{1}(0, \infty)+L_{\infty}(0, \infty)$, where $f \prec g$ means that for every $0<s<\infty$

$$
\int_{0}^{s} f^{*}(t) d t \leq \int_{0}^{s} g^{*}(t) d t
$$

Here $f^{*}(t)$ is the decreasing rearrangement of $|f(t)|$. Lemma 1 is reformulated for this situation in the obvious way (see also Lemma 3 and Remark 2 below). Instead of a partition of $N$ into disjoint subsets $C_{k}$ and bijections $\pi_{k}$ we use a partition of $(0, \infty)$ into an infinite family of disjoint subsets of infinite measure and measure preserving transformations.

COROLlARY 2. Let $\Phi$ be an Orlicz function, and let $L_{\Phi}(0, \infty)$ be the corresponding Orlicz space equipped with the Luxemburg norm. Then the following conditions are equivalent:

(1) $\Phi$ satisfies the $\Delta_{2}$-condition; 
(2) $L_{\Phi}(0, \infty) \in(\mathrm{UHlm})$.

PROOF. By [1] the $\Delta_{2}$ condition for $\Phi$ is equivalent to (UMB) property for $L_{\Phi}(0, \infty)$.

The Banach lattice $E$ is said to satisfy a lower q-estimate if there exists a constant $C>0$ such that for all finite sequence $\left(x_{n}\right)$ of mutually disjoint elements in $E$

$$
\left(\sum_{n}\left\|x_{n}\right\|_{E}^{q}\right)^{1 / q} \leq C\left\|\sum_{n} x_{n}\right\|_{E} .
$$

Combining Theorem 3 and Corollary 2.11 [3] we obtain the following:

COROLLARY 3. If $E$ is a symmetric function space on $(0, \infty)$, then $E$ satisfies a lower $q$-estimate for some $1<q<\infty$ if and only if there is an equivalent symmetric norm $\|\cdot\|_{0}$ on $E$ such that $\left(E,\|\cdot\|_{0}\right) \in(\mathrm{UMB})$.

\section{3. (UH $\mu)$-property for the symmetric operator spaces $E(M, \mu)$}

In this section $(M, \mu)$ will be a semifinite von Neumann algebra $M$ with a faithful semifinite normal trace $\mu$ on $M$. Let $K(M, \mu)$ denote the space of all $\mu$-measurable operators affiliated with $M$ (see [5]). $K(M, \mu)$ is the closure of $M$ with respect to the measure topology generated by the trace $\mu$ with fundamental system of neighbourhoods around 0 given by $V(\epsilon, \delta)=\{T \in K(M, \mu)$; there exists a projection $P$ in $M$ such that $\|T P\|_{\infty} \leq \epsilon$ and $\left.\mu(1-P) \leq \delta\right\}$ for $\epsilon, \delta>0$. Here 1 is the unit of $M$ and $\|\cdot\|_{\infty}$ is the $C^{*}$-norm on $M$. We shall denote by $x_{n} \stackrel{\mu}{\longrightarrow} x$ the convergence of the sequence $\left(x_{n}\right)$ to $x$ in the measure topology generated by the trace $\mu$. Let $A \in K(M, \mu)$. The $t$-th singular number of $A \mu_{t}(A)$ is

$$
\mu_{t}(A)=\inf \left\{\|A P\|_{\infty}: P \text { is a projection in } M \text { with } \mu(1-P) \leq t\right\}, t>0
$$

(see, for example [5, Definition 2.1]). It is known [5] that $\mu_{t}(A)=\mu_{t}\left(A^{*}\right)=\mu_{t}(|A|)$ where $|A|=\left(A^{*} A\right)^{1 / 2}$.

Let $E(0, \mu(1))$ be a separable symmetric function space. The symmetric operator space $E(M, \mu)$ is the space of operators $A \in K(M, \mu)$ such that $\mu_{t}(A)$ belongs to $E(0, \mu(1))$ and

$$
\|A\|_{E(M, \mu)}=\|\mu(A)\|_{E(0, \mu(1))} .
$$

Before formulating the main result of this section which concerns the (UH $\mu$ ) property, we note that if $E(M, \mu) \in(\mathrm{UH} \mu)$ then $E(M, \mu)$ possesses the Kadec-Klee property with respect to measure convergence (notation: $E(M, \mu) \in(\mathrm{H} \mu)$ ). In 
the setting of symmetric function spaces, the property $(\mathrm{Hm})$ has been investigated

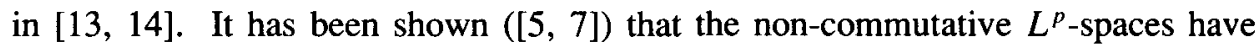
property $(\mathrm{H} \mu)$; subsequently, it was proved in [16] that $E(0, \mu(1)) \in(\mathrm{Hm})$ implies $E(M, \mu) \in(\mathrm{Hm})$. Further, it has been established in [16] that if $E(0, \mu(1))$ is an arbitrary separable symmetric space then $E(M, \mu)$ can be renormed equivalently so that $E(M, \mu)$ endowed with the new norm $\|\cdot\|^{\prime}$ is a symmetric operator space and $\left(E(M, \mu),\|\cdot\|^{\prime}\right) \in(\mathrm{H} \mu)$.

The main result of this section shows the uniform Kadec-Klee property with respect to convergence in measure extends from the symmetric function space $E(0, \mu(1))$ to $E(M, \mu)$.

THEOREM 4. If $E(0, \mu(1)) \in(\mathrm{UHm})$, then $E(M, \mu) \in(\mathrm{UH} \mu)$.

The proof of Theorem 4 is based mainly on the following result (cf. [9, Theorem 2.1]).

LEMMA 2. Let $M, \mu, E(0, \mu(1)), E(M, \mu)$ be as above, and let $\left(x_{n}\right) \subseteq E(M, \mu)$, satisfy $x_{n} \stackrel{\mu}{\longrightarrow} 0$. There exist two sequences of pairwise orthogonal projections $\left(p_{k}\right),\left(q_{k}\right) \subseteq M$ and subsequence $\left(x_{n_{k}}\right)$ such that

$$
\left\|x_{n_{k}}-q_{k} x_{n_{k}} p_{k}\right\|_{E(m, \mu)} \rightarrow 0 .
$$

We also need the following non-commutative analog of Lemma 1 (see also proof of the implication (iii) $\rightarrow$ (i) of Theorem 3).

LEMMA 3. Let $a, b, c, d \in E(M, \mu)$ be positive operators such that $\mu_{t}(b)=\mu_{t}(c)$, $\mu_{t}(a) \leq \mu_{t}(d)$ for all $t \geq 0$ and $a c=0$. Then

$$
\|a+c\|_{E(M, \mu)} \leq\|d+b\|_{E(M, \mu)} .
$$

PROOF OF LEMMA 3. Let $x, y \in K(M, \mu)$. The notation $x \prec y$ means

$$
\int_{0}^{t} \mu_{\tau}(x) d \tau \leq \int_{0}^{t} \mu_{\tau}(y) d \tau
$$

for all $t>0$. Since $E(0, \mu(1))$ is a separable symmetric space the relation $x \prec y, y \in$ $E(M, \mu)$ implies $x \in E(M, \mu)$ and $\|x\|_{E(M, \mu)} \leq\|y\|_{E(M, \mu)}$ (see, for example [16]). So, it suffices to prove that $a+c \prec d+b$. Fix $t>0$. Without loss of generality we can assume that $M$ has no minimal projections and therefore there are projections $P_{1}, P_{2} \in M$ such that $P_{1} P_{2}=0, \mu\left(P_{1}+P_{2}\right)=t$ and $\int_{0}^{t} \mu_{\tau}(a+c) d \tau=\mu\left(a P_{1}+c P_{2}\right)$ (see [5, Lemma 4.1 and subsequent remarks]). Since $\mu_{t}(c)=\mu_{t}(b), \mu_{t}(a) \leq \mu_{t}(d)$ we can find the projections $P_{3}, P_{4} \in M$ such that $\mu\left(P_{3}\right)=\mu\left(P_{2}\right), \mu\left(P_{4}\right)=\mu\left(P_{1}\right)$ 
and $\mu\left(b P_{3}\right)=\mu\left(c P_{2}\right), \mu\left(a P_{1}\right) \leq \mu\left(d P_{4}\right)$. Put $P_{5}=P_{4} \vee P_{3}$. Then $\mu\left(P_{5}\right) \leq t$ and it follows that

$$
\begin{aligned}
\int_{0}^{t} \mu_{\tau}(d+b) d \tau & \left.\geq \mu\left((d+b) P_{5}\right) \geq \mu\left(d P_{4}\right)+\mu\left(b P_{3}\right)\right) \\
& \geq \mu\left(a P_{1}+c P_{2}\right)=\int_{0}^{t} \mu_{\tau}(a+c) d \tau
\end{aligned}
$$

REMARK. Lemma 1, and its continuous analog in the implication (iii) $\rightarrow$ (i) of Theorem 3, follow from Lemma 3 as particular cases.

PRoOF of TheOREM 4. Suppose that $x_{n}, x \in E(M, \mu),\left\|x_{n}\right\|_{E(M, \mu)}=1, \| x_{n}-$ $x_{m} \|_{E(M, \mu)} \geq \epsilon(m \neq n)$, and $x_{n} \stackrel{\mu}{\longrightarrow} x$. We can assume that $x \neq 0$. Put $x_{n}=x+y_{n}$. It is clear that $y_{n} \stackrel{\mu}{\longrightarrow} 0$ and $\left\|y_{n}-y_{m}\right\|_{E(M, \mu)} \geq \epsilon$. By Lemma 2 we may assume by passing to a subsequence, if necessary, that there exist $\left(p_{n}\right),\left(q_{n}\right) \subset M$ such that $p_{n}=p_{n}^{*}=p_{n}^{2}, q_{n}=q_{n}^{*}=q_{n}^{2}$, for all $n=1,2, \ldots, p_{n} p_{m}=q_{n} q_{m}=0(n \neq m)$ and $\left\|y_{n}-p_{n} y_{n} q_{n}\right\|_{E(M, \mu)} \rightarrow 0$. Put $P_{n}=\bigvee_{i=n}^{\infty} p_{i}, Q_{n}=\bigvee_{i=n}^{\infty} q_{i}$. It is evident that $P_{n} \downarrow 0, Q_{n} \downarrow 0$ and hence $P_{n}^{\perp} \uparrow 1, Q_{n}^{\perp} \uparrow 1$. Our first objective is to show that

$$
\left\|x-P_{n}^{\perp} x Q_{n}^{\perp}\right\|_{E(M, \mu)} \rightarrow 0
$$

for all $x \in E(M, \mu)$. Indeed, without loss of generality we can assume that $x \geq$ 0 . Since $x=P_{n}^{\perp} x Q_{n}^{\perp}+P_{n} x Q_{n}^{\perp}+P_{n}^{\perp} x Q_{n}+P_{n} x Q_{n}$ it is sufficient to prove that $\left\|P_{n}^{\perp} x Q_{n}\right\|_{E(M, \mu)},\left\|P_{n}^{\perp} x Q_{n}\right\|_{E(M, \mu)},\left\|P_{n} x Q_{n}\right\|_{E(M, \mu)} \rightarrow 0$.

By ([16, Lemma 3]), we have

$$
\left\|P_{n}^{\perp} x Q_{n}\right\|_{E(M, \mu)}=\left\|P_{n}^{\perp} \sqrt{x} \sqrt{x} Q_{n}\right\|_{E(M, \mu)} \leq\left\|\sqrt{x} P_{n}^{\perp} \sqrt{x}\right\|_{E(M, \mu)}^{1 / 2}\left\|Q_{n} x Q_{n}\right\|_{E(M, \mu)}^{1 / 2} .
$$

Since

$$
\begin{gathered}
\left\|\sqrt{x} P_{n}^{\perp} \sqrt{x}\right\|_{E(M, \mu)}=\left\|P_{n}^{\perp} x P_{n}^{\perp}\right\|_{E(M, \mu)} \leq\|x\|_{E(M, \mu)} \text { and } \\
\left\|Q_{n} x Q_{n}\right\|_{E(M, \mu)} \rightarrow 0
\end{gathered}
$$

([16, Proposition 4]) we have $\left\|P_{n}^{\perp} x Q_{n}\right\|_{E(M, \mu)} \rightarrow 0$. Similarly, $\left\|P_{n} x Q_{n}^{\perp}\right\|_{E(M, \mu)}$, $\left\|P_{n} x Q_{n}\right\|_{E(M, \mu)} \rightarrow 0$.

Now, using (2) and the fact that $\left\|y_{n}-p_{n} y_{n} q_{n}\right\|_{E(M, \mu)} \rightarrow 0$, we may assume by passing to a subsequence and relabelling if necessary, that

$$
x_{n}=P_{n}^{\perp} x Q_{n}^{\perp}+p_{n} y_{n} q_{n}+z_{n}
$$

where $\left\|z_{n}\right\|_{E(M, \mu)} \leq \epsilon 2^{-n}$. 
Fix $\epsilon>0$. Now let $\delta=\delta\left(2^{-1} \epsilon\right)$ be chosen as in the definition of (UHm) for $E(0, \mu(1))$, and let the integer $N$ simultaneously satisfy the inequalities

$$
\begin{aligned}
\left\|x-P_{N}^{\perp} x Q_{N}^{\perp}\right\|_{E(M, \mu)} & <\delta 2^{-N}, \\
\left\|P_{N}^{\perp} x Q_{N}^{\perp}\right\|_{E(M, \mu)} & \geq 2^{-1}\|x\|_{E(M, \mu)} .
\end{aligned}
$$

Divide $(0, \mu(1))$ into an infinite family of disjoint subsets $\left(A_{i}\right)_{i=N}^{\infty}$ such that $m\left(A_{N}\right)=$ $\mu\left(Q_{N}^{\perp}\right), m\left(A_{i}\right)=\mu\left(q_{i}\right), \quad i=N+1, \ldots$ and choose sequence $\left(f_{i}(t)\right)_{i=N}^{\infty} \subset$ $E(0, \mu(1))$ such that $f_{i}(t) \chi_{A_{i}}(t)=f_{i}(t)$ for all $i \geq N$ and $f_{N}^{*}(t)=\mu_{t}\left(P_{N}^{\perp} x Q_{N}^{\perp}\right)$, $f_{i}^{*}(t)=\mu_{t}\left(p_{i} y_{i} q_{i}\right), i \geq N$. Notice that for $n \geq N$

$$
\begin{aligned}
\left|P_{N}^{\perp} x Q_{N}^{\perp}+p_{n} y_{n} q_{n}\right|^{2} & =\left(Q_{N}^{\perp} x^{*} P_{N}^{\perp}+q_{n} y_{n}^{*} p_{n}\right)\left(P_{N}^{\perp} x Q_{N}^{\perp}+p_{n} y_{n} q_{n}\right) \\
& =\left|P_{N}^{\perp} x Q_{N}^{\perp}\right|^{2}+\left|p_{n} y_{n} q_{n}\right|^{2} .
\end{aligned}
$$

Hence $\left|P_{N}^{\perp} x Q_{N}^{\perp}+p_{n} y_{n} q_{n}\right|=\left|P_{N}^{\perp} x Q_{N}^{\perp}\right|+\left|p_{n} y_{n} q_{n}\right|$, and therefore

$$
\mu_{t}\left(P_{N}^{\perp} x Q_{N}^{\perp}+p_{n} y_{n} q_{n}\right)=\left(f_{N}+f_{n}\right)^{*}(t)
$$

for all $t>0$ and for all $n \geq N$. Using (6) it then follows that for $n \geq N$,

$$
\left\|f_{N}+f_{n}\right\|_{E(0, \mu(1))}=\left\|P_{N}^{\perp} x Q_{N}^{\perp}+p_{n} y_{n} q_{n}\right\|_{E(M, \mu)} .
$$

Since $P_{n}^{\perp} \geq P_{N}^{\perp}, Q_{n}^{\perp} \geq Q_{N}^{\perp}$ for $n \geq N$ we have $\mu_{t}\left(P_{N}^{\perp} x Q_{N}^{\perp}\right)=\mu_{t}\left(P_{N}^{\perp} P_{n}^{\perp} x Q_{n}^{\perp} Q_{N}^{\perp}\right) \leq$ $\mu_{t}\left(P_{n}^{\perp} \times Q_{n}^{\perp}\right)$ for all $t \geq 0$ (see [5]) and hence by Lemma 3

$$
\begin{aligned}
\left|P_{N}^{\perp} x Q_{N}^{\perp}+p_{n} y_{n} q_{n}\right| & =\left|P_{N}^{\perp} x Q_{N}^{\perp}\right|+\left|p_{n} y_{n} q_{n}\right| \prec\left|P_{n}^{\perp} x Q_{n}^{\perp}\right|+\left|p_{n} y_{n} q_{n}\right| \\
& =\left|P_{n}^{\perp} x Q_{n}^{\perp}+p_{n} y_{n} q_{n}\right| .
\end{aligned}
$$

It follows that $\left|P_{N}^{\perp} x Q_{N}^{\perp}+p_{n} y_{n} q_{n}\right|_{E(M, \mu)} \leq\left\|P_{n}^{\perp} x Q_{n}^{\perp}+p_{n} y_{n} q_{n}\right\|_{E(M, \mu)}$ and so, by (7) and (3)

$$
\left\|f_{N}+f_{n}\right\|_{E(0, \mu(1))} \leq 1+\epsilon 2^{-n} \text { for } n>N .
$$

Using (5) and passing to a subsequence if necessary we may assume that $\| f_{N}+$ $f_{n} \|_{E(0, \mu(1))} \rightarrow \alpha$, where $2^{-1}\|x\|_{E(M, \mu)} \leq \alpha \leq 1$. It follows that $\left\|\beta_{n}^{-1}\left(f_{N}+f_{n}\right)\right\|_{E(0, \mu(1))}=$ 1 and

$$
\left\|\beta_{n}^{-1}\left(f_{N}+f_{n}\right)-\beta_{m}^{-1}\left(f_{N}+f_{m}\right)\right\|_{E(0, \mu(1))} \geq 2^{-1} \epsilon
$$

for sufficiently large $m, n$, where $\beta_{n}=\left\|f_{N}+f_{n}\right\|_{E(0, \mu(1))}$ (the last inequality is the simple consequence of the following correlations

$$
\begin{aligned}
& \left\|p_{n} y_{n} q_{n}-y_{n}\right\|_{E(M, \mu)} \rightarrow 0, \quad\left\|p_{n} y_{n} q_{n}-p_{m} y_{m} q_{m}\right\|_{E(M, \mu)}=\left\|f_{n}-f_{m}\right\|_{E(0, \mu(1))}, \\
& \left.\quad \beta_{n} \rightarrow \alpha \text { and }\left\|y_{n}-y_{m}\right\|_{E(M, \mu)} \geq \epsilon\right) .
\end{aligned}
$$


Observe that $p_{n} y_{n} q_{n} \stackrel{\mu}{\longrightarrow} 0$ implies $f_{n} \stackrel{m}{\longrightarrow} 0$ and therefore $\beta_{n}^{-1}\left(f_{N}+f_{n}\right) \stackrel{m}{\longrightarrow}$ $\alpha^{-1} f_{N}$. So, $E(0, \mu(1)) \in(\mathrm{UHm})$ implies $\left\|\alpha^{-1} f_{N}\right\|_{E(0, \mu(1))}<1-\delta$. It follows that $\left\|P_{N}^{\perp} x Q_{N}^{\perp}\right\|_{E(M, \mu)}=\left\|f_{N}\right\|_{E(0, \mu(1))} \leq 1-\delta$. Then, by (4), $\|x\| \leq 1-\delta+\delta 2^{-N}$. This completes the proof of Theorem 4 .

The following corollary extends results of $[5,7]$ which assert $L_{p}(M, \mu) \in(\mathrm{H} \mu)$ for all $p \geq 1$.

COROllaRY 4. If $\Phi \in \Delta_{2}$, then $L_{\Phi}(M, \mu) \in(\mathrm{UH} \mu)$.

The proof immediately follows from Corollary 2 and Theorem 4.

COROLlARY 5. If $\phi(t), \phi(0)=0$, is a concave, increasing function on $(0, \mu(1))$ such that $\phi(\infty)=\infty$ if $\mu(1)=\infty$, then the Lorentz space $\Lambda_{\phi}(M, \mu)$ has the uniform Kadec-Klee property with respect to convergence in measure.

PROOF. By Theorem 4 it is sufficient to prove that $\Lambda_{\phi}(0, \mu(1)) \in(\mathrm{UHm})$. But this is easy follows from the results $[14,15]$ which assert that if $y \stackrel{m}{\longrightarrow} 0, y_{n} \in \Lambda_{\phi}(0, \mu(1))$ then

$$
\left\|x_{n}+y_{n}\right\|_{\Lambda_{\phi}(0, \mu(1))}=\|x\|_{\Lambda_{\phi}(0, \mu(1))}+\left\|y_{n}\right\|_{\Lambda_{\phi}(0, \mu(1))}+o(1) .
$$

\section{References}

[1] M. A. Akcoglu and L. Sucheston, 'La monotonicité uniforme des normes et théorèmes ergodiques', C. R. Acad. Sc. Paris, t. 301, Serie I, N 7 (1985), 359-360.

[2] G. Birkhoff, Lattice theory, A.M.S. Colloquium Publications, XXV, 3rd edition, (Amer. Math. Soc., Providence, 1967).

[3] P. G. Dodds, T. K. Dodds, P. N. Dowling, C. J. Lennard and F. A. Sukochev, 'A uniform Kadec-Klee property for symmetric operator spaces', Math. Proc. Cambridge Philos. Soc., to appear.

[4] D. van Dulst and V. de Valk, ' $(K K)$ properties, normal structure and fixed points of nonexpansive mapping in Orlicz sequence spaces', Canad. J. Math. 38 (1986), 728-750.

[5] T. Fack and H. Kosaki, 'Generalized $s$-numbers of $\tau$-measurable operators', Pacific J. Math. 123 (1986), 269-300.

[6] Y.-P. Hsu, 'The lifting of the $U K K$ property from $E$ to $C_{E}$ ', (1993), preprint.

[7] H. Kosaki, 'Applications of uniform convexity of noncommutative $L^{p}$-spaces', Trans. Amer. Math. Soc. 283 (1984), 265-282.

[8] S. G. Krein, Ju. I. Petunin and E. M. Semenov, Interpolation of linear operators, Translation of Mathematical Monographs 54 (Amer. Math. Soc., 1982).

[9] A. V. Krygin, F. A. Sukochev and V. E. Sheremetjev, 'Convergence by measure, weak convergence and structure of subspaces in the symmetric spaces of measurable operators', Dep. VINITI N2487B92 , 1-34 (Russian). 
[10] J. Lindenstrauss and L. Tzafriri, Classical Banach spaces, I. Sequence spaces (Springer, Berlin, 1977).

[11] _ Classical Banach spaces, II. Function spaces (Springer, Berlin, 1979).

[12] S. Y. Novikov, 'Type and cotype of Lorentz function spaces', Mat. zametki 32 (2) (1982), 213-221.

[13] W. P. Novinger, 'Mean convergence in $L^{p}$-spaces', Proc. Amer. Math. Soc. 34 (1972), 627-628.

[14] A. A. Sedaev, 'On $(H)$-property in the symmetric spaces', Teoriya funkcii, funkc. anal. i prilozenia 11 (1970), 67-80 (Russian).

[15] _ ' 'On weak and norm convergence in interpolation spaces', Trudy 6 zimney shkoly po mat. programm. i smezn. voprosam, Moskow (1975), 245-267 (Russian).

[16] F. A. Sukochev and V. I. Chilin, 'Convergence in measure in admissible non-commutative symmetric spaces', Izv. Vyss. Uceb. Zaved. 9 (1990), 63-70 (Russian).

[17] M. Takesaki, Theory of operator algebras I (Springer-Verlag, New York, 1979).

Department of Mathematics and Statistics

The Flinders University

G.P.O. Box 2100

Adelaide, SA 5001

Australia

e-mail: sukochev@ist.flinders.edu.au 